\title{
HIL Evaluation of a Novel Real-time Energy Management System for an HEV with a Continuously Variable Transmission
}

\author{
Amir Taghavipour* - Ali Alipour \\ K. N. Toosi University of Technology, Faculty of Mechanical Engineering, Iran
}

One of the most important challenges facing automotive engineers is reducing vehicle fuel consumption and improving the drivability index. Modern hybrid electric powertrains play an important role in reducing fuel consumption. Continuously variable transmission (CVT) is an automatic transmission that can change the gear ratio seamlessly using a belt and pulleys. CVT performs with infinite gear ratios. Controlling and determining the optimal gear ratio, especially in a complex hybrid powertrain, is a major challenge. Therefore, a multi-parametric model predictive controller with real-time implementation capability is proposed that can handle the energy management task concurrently with gear shifting strategy in a parallel pre-transmission hybrid electric vehicle. The proposed controller hardware-in-the-loop (HIL) validation procedure on the high-fidelity Autonomie model shows significant improvement in fuel economy while maintaining drivability in three different driving schedules. HIL evaluation guarantees the real-time capability and the proposed controller readiness to be implemented to real-world control hardware.

Keywords: hybrid electric vehicle, continuously variable transmission (CVT), Autonomie, energy management system, explicit model predictive control, hardware in the loop.

\begin{abstract}
Highlights
- Proposing a novel control-oriented model for hybrid electric powertrain with a continuously variable transmission.

- Developing an optimal energy management system via an explicit model predictive control approach.

- Hardware-in-the-loop testing via an experimentally-validated high-fidelity model to represent the real-time capability of the proposed controller.
\end{abstract}

\section{INTRODUCTION}

With the growing environmental concerns about global warming and shortages of global fuel reserves, every possible solution to reduce transportation energy consumption has become a key element in researchers' current debate. Vehicles' fuel consumption plays a crucial role in determining the emission of greenhouse gases from the transportation sector. As a result, vehicles have been increasingly facing stringent emissions and fuel economy standards, which are driven by regulatory and market forces. Among the proposed solutions to improve the efficiency of conventional powertrains, continuously variable transmission (CVT) has been commercialized. A CVT changes gear ratios seamlessly between desired limits, which consequently enhances the fuel economy and dynamic performance of a vehicle by better matching the engine operating conditions to the variable driving scenarios.

In contrast, powertrain electrification has also proven to be an effective solution to the aforementioned problems. New opportunities are expected to appear by using CVT with a hybrid electric powertrain, although the controls design complexity can be a potential drawback. For those hybrid architectures whose internal combustion engine is connected to the wheels, a separate transmission controls the engine operation. Except for the series powertrains, the internal combustion engine (ICE) is controlled mechanically or electrically. For complex hybrid structures, a second electric machine (apart from the traction electric motor) plays a pivotal rule in controlling the engine. Since those drivetrains theoretically provide infinite ranges of imaginary gear ratios, they are called electrical continuously variable transmission (eCVT). However, in most commercialized parallel architectures, a mechanical gearbox such as automatic or dualclutch transmission regulates the engine status. By replacing those conventional transmissions with a CVT, more flexibility for fuel economy and emissions improvement is provided. However, a sophisticated control scheme should be available to exploit the mentioned flexibility fully.

Among different approaches for control design, model-based methods seem to be promising. In contrast to heuristic methods, they can reduce the time and cost of the control development procedure, and their performance and optimality can be guaranteed for different scenarios facing the powertrain. One of the challenges of using model-based approaches is deriving an appropriate control-oriented model that is simultaneously simple and adequately accurate. 
The accuracy of the control-oriented model in terms of capturing the essential dynamics of the system contributes to the controller's performance. However, the higher levels of accuracy require more dynamics and, consequently, larger models. These larger models may require more computational time and complexity, which are contradicting factors in the real-time implementation of the controller. Therefore, we should keep the control-oriented model simple enough to maintain the controller's computational burden at a very low level. This trade-off turns out to be the most demanding bottleneck of designing a model-based controller. Acquiring such a model along with a model-based optimal controller that can be implemented in real-time remains a challenge, which we focus on in this research. The development of an optimum CVT control strategy is not an easy task, owing to two partially opposite features that have to be satisfied: The reduction of fuel consumption and the requirement of appropriate drivability/acceleration performance which is dependent on the torque capacity of the CVT system. Accurate and fast control of the rate of speed ratio change is a prerequisite for meeting these goals. Pfiffner and Guzzella [1] have investigated the optimal fuel consumption performance of a vehicle equipped with a CVT under steady and transient conditions. Three basic modes for optimal control are introduced, and then the optimal performance conditions are calculated by defining the optimization problem to reduce specific fuel consumption, inputs of load and pedal functions and gear ratio change. The results showed that the optimization procedure improved fuel economy by 4 $\%$ for the supercharged engine and $6 \%$ for the sparkignition engine. Hatakana et al. [2] presented the receding horizon control strategy and introduced this controller as a new control scheme without the need for a control map in order to determine the gear ratio for the CVT system. For this purpose, two spraying and non-spraying modes were considered, and the equations of these two engine states were obtained. Kazemi et al. [3] investigated the optimal gear ratios for the modelled CVT system using nonlinear optimal control rules. They presented a model of the power transmission system and its subsystems. Then, by defining the optimization problem, the optimal gear ratios were calculated, and a fuel consumption reduction of $11 \%$ was reported. However, the realtime implementation issues remained unaffected. Yildiz and Kopmaz [4] have carefully studied the dynamics of CVT and developed a control-oriented model, which has been validated via a test rig in order to design appropriate controller for shifting.
Bemporad et al. [5] have proposed a supervisory controller for a powertrain with a CVT transmission, which is scheduled for production by General Motors. The control strategy is an online model predictive control (MPC) that determines the engine torque and CVT track the demanded torque by the driver for improving the fuel economy. Based on extensive invehicle testing, the supervisory controller proved to be robust to different environmental conditions and driving manoeuvres. Tao et al. [6] have proposed and hardware-in the loop (HIL) validated control strategy for a CVT transmission and shown the sensitivity of throttle opening and vehicle speed to ratio control; consequently, its fluctuation can be reduced dramatically. All those achievements are contributing to efficiency improvement and jerk reduction. In addition to those studies, various investigations have been carried out to control this gearbox by using various controls, such as proportional-integralderivative (PID) [7], linear-quadratic regulator (LQR) [8], fuzzy control [9], backstepping control [10].

As for CVT-based hybrid vehicles, Hofman et al. [11] investigated the sizing problem of electric machine for a hybrid vehicle equipped with a CVT regarding the best fuel economy. By studying the parameters of the model, they have developed a quick simulation platform, which is suitable for control design purposes. Ji et al. [12] have proposed a realtime energy management strategy for a CVT-based parallel hybrid electric vehicle. By using AVL-Cruise software as their simulation platform, they could improve the fuel economy by $1.9 \%$ based on an urban dynamometer driving schedule (UDDS). Tang et al. [13] have proposed a Gauss pseudo-spectral MPC strategy for a parallel CVT-based HEV and validated the scheme on a new European drive cycle (NEDC), which shows promising improvement in fuel economy. Li et al. [14] have proposed a strategy for such a powertrain by using the rules from the dynamic programming solution. They also extend their studies on the equivalent consumption minimization strategy (ECMS) implementation on CVT-based HEV and compared the resulted fuel economy based on different ultimate battery states of charge for different drive cycles [15]. Similar studies have been conducted recently for CVT-based plugin hybrid electric vehicles (PHEVs), which admits the growing attention to find an ultimate solution for controls development. Wang and Frank proposed a control scheme for a CVT-based PHEV indicates that in order to achieve the global optimum, energy management system (EMS) and gear shifting strategy should be done simultaneously [16]. Li et al. [14] have 
studied multiple optimal gear-shifting strategies by considering hydraulics and mechanical loss of CVT for a CVT-based PHEV.

The challenge faced by this research is to obtain a simple and adequately accurate control-oriented model for a real-time implementable model-based controller that can handle energy management and CVT gear shifting at the same time. To outperform the other proposed controllers, this approach should be optimal with a minimal computational burden. Thus, we propose a multi-parametric model predictive approach to develop an energy management system for a CVT-based parallel HEV to improve fuel economy further, while maintaining drivability indices and, more importantly, real-time implementation capability of the controller. This controller performance will be validated with the dSPACE HIL platform along with high-fidelity experimentally-validated Autonomie model of the mentioned powertrain through three different driving cycles. The results suggest a considerable improvement in fuel economy compared to the baseline controller and mentioned studies, while the HIL evaluation guarantees the real-time capability and the proposed controller readiness to be implemented to real-world control hardware.

First, the control-oriented model is introduced. Then a brief description of the high-fidelity Autonomie model whose parameters are derived based on the experimental data is delivered. In Section 2, the control design procedure is detailed. and then a brief specification of the HIL platform is presented. Next, the results of the simulation are discussed. Section 5 concludes the article.

\section{MODELLING}

The performance of a model-based controller is highly dedicated to the dynamics that are included inside it. However, an accurate model can be of higher orders, which imposes a significant computational load on the controller. Thus, the model should be sufficiently simple and accurate to be useful for the development of controls. The trade-off between simplicity and accuracy of the control-oriented model determines the performance and real-time capability of the controller. During the controller development procedure, it should go through multiple virtual simulation stages until it can be qualified to be implemented on the real plant. To this end, a valid simulation platform should be provided at the early stages, so that the controller performance can be tested beforehand, and its issues and flows are revisited and resolved. In this section, we introduce the control-oriented model of the powertrain and then review the experimentallyvalidated simulation model that is to be used in our HIL procedure.

\subsection{Control-oriented Model}

As mentioned above, acquiring a simple yet sufficiently accurate control-oriented model is an important step in designing a model-based controller. Here, we introduce a control-oriented model that represents the complexity of the hybrid powertrain, but it only contains the essential dynamics of it according to Eq. (1). The first equation determines the battery dynamics in its simplest shape. The high-voltage battery state of charge (SOC) will be determined by the current drawn from it and its total capacity. Since the traction motor torque is approximately proportional to its current, we can find a way to rewrite this equation with traction motor torque $\left(\left(T_{m}=k_{m} I\right)\right)$. The second equation is based on the vehicle longitudinal dynamics. In this equation, the engine, traction motor, and brake torque along with CVT ratio determine the variation of the vehicle velocity.

$$
\left\{\begin{array}{rl}
S \dot{O} C & =-\frac{I}{Q}=-\frac{T_{m}}{k_{m} Q} \\
\dot{V} & =\frac{1}{m r_{w}}\left\{\eta_{c v t} \eta_{D L} i_{F D} i_{c v t}\left(T_{m}+T_{e}\right)-T_{b}\right\} \\
& -\frac{\rho c_{D} A_{f} V^{2}}{2 m}-f_{r} g
\end{array},\right.
$$

where $T_{m}, T_{e}$ and $T_{b}$ are the electric motor, engine, and mechanical brake torque, respectively along with $i_{c v t}$ as the CVT ratio constitute the manipulated inputs. SOC and $V$ are battery state of charge and vehicle velocity that are the problem state variables. The incorporated parameters are as follows: $Q, k_{m}, m, \delta, r_{w}, \eta_{c v t}, \eta_{D L}, i_{F D}$, $\rho, c_{D}, A_{f}, f_{r}$, and $g$ are battery capacity, electric motor coefficient, vehicle mass, rotational inertias equivalent coefficient, wheel effective radius, CVT efficiency, driveline efficiency, final drive ratio, air density, drag coefficient, vehicle frontal area, rolling friction coefficient and gravity constant, respectively.

Although the original nature of these equations is nonlinear, according to Eq. (1), we can approximate them with their linear counterparts around any possible operating point (Eq. (2)). 


$$
\begin{aligned}
& {\left[\begin{array}{c}
S \dot{O O C} \\
\dot{V}
\end{array}\right]=\left[\begin{array}{cc}
0 & 0 \\
0 & -\frac{\rho c_{D} A_{f} \hat{V}}{2 m}
\end{array}\right]\left[\begin{array}{c}
S O C \\
V
\end{array}\right]} \\
& +\left[\begin{array}{cccc}
-\frac{1}{k_{m} Q} & 0 & 0 & 0 \\
\frac{\eta_{c v t} \eta_{D L} i_{F D} \hat{i}_{c v t}}{m r_{w}} & \frac{\eta_{c v t} \eta_{D L} i_{F D} \hat{i}_{c v t}}{m r_{w}} & \frac{\eta_{c v v} \eta_{D L} i_{F D}}{m r_{w}}\left(\hat{T}_{m}+\hat{T}_{e}\right) & -\frac{1}{m r_{w}}
\end{array}\right]\left[\begin{array}{c}
T_{m} \\
T_{e} \\
i_{c v t} \\
T_{b}
\end{array}\right] \\
& +\left[\begin{array}{c}
0 \\
\frac{\eta_{c v t} \eta_{D L} i_{F D} \hat{i}_{c v t}}{m r_{w}}\left(\hat{T}_{m}+\hat{T}_{e}\right)+\frac{\rho c_{D} A_{f} \hat{V}^{2}}{2 m}-f_{r} g
\end{array}\right],
\end{aligned}
$$

where $\hat{V}, \hat{T}_{m}, \hat{T}_{e}$, and $\hat{i}_{c v t}$ are the state variable and input at any operating point. The parameters values are listed in Table 1.

\subsection{Simulation Model}

A schematic of the CVT-based parallel hybrid electric vehicle in Autonomie software is shown in Fig 1. The advantage of the Autonomie platform over similar softwares is that it offers a sophisticated model of different powertrains derived from Argonne National Lab test procedures and its capability to be used for performing HIL tests.

According to Fig. 1, the internal combustion engine (ICE) is connected to a traction motor (MOT), which is powered by the high-voltage battery (HVB) via an electrical coupling (ED). Then the output shaft of the power plant is connected to the CVT. The CVT magnifies the torque and transfers it to the final drive (FD) via a mechanical coupling $(\mathrm{CP})$, which drives the vehicle (VM) by the wheels (WH). The vehicle accessories load (ACC) is also considered

\begin{tabular}{|c|c|c|}
\hline Parameter & Description & Value \\
\hline$Q$ & Battery capacity [Ah] & 6.53 \\
\hline$k_{m}$ & Motor torque constant $[\mathrm{N} \cdot \mathrm{m} / \mathrm{A}]$ & 0.04 \\
\hline$m$ & Vehicle mass [kg] & 1700 \\
\hline$r_{w}$ & Wheel effective radius [m] & 0.35 \\
\hline$\eta_{c v t}$ & CVT efficiency & 0.95 \\
\hline$\eta_{D L}$ & Driveline efficiency & 0.9 \\
\hline$i_{F D}$ & Final drive ratio & 6.39 \\
\hline$\rho$ & Air density $\left[\mathrm{kg} / \mathrm{m}^{3}\right]$ & 1.4 \\
\hline$c_{D}$ & Drag coefficient & 0.35 \\
\hline$A_{f}$ & Vehicle frontal area [m²] & 3.2 \\
\hline$f_{r}$ & Rolling resistance coefficient & 0.01 \\
\hline$g$ & Gravity acceleration $\left[\mathrm{m} / \mathrm{s}^{2}\right]$ & 9.81 \\
\hline$T_{m, \max }$ & Motor maximum torque [Nm] & 160 \\
\hline$T_{m, \min }$ & Motor minimum torque [Nm] & -160 \\
\hline$T_{e, \max }$ & Engine maximum torque [Nm] & 207 \\
\hline$T_{e, \min }$ & Engine minimum torque [Nm] & 0 \\
\hline$i_{c v t, \max }$ & CVT maximum ratio & 2.5 \\
\hline$i_{c v t, \min }$ & CVT minimum ratio & 0.5 \\
\hline$T_{b, \max }$ & Brake maximum torque [Nm] & 6000 \\
\hline$T_{b, \min }$ & Brake minimum torque [Nm] & 0 \\
\hline
\end{tabular}
in the vehicle model. The engine model consists of
Table 1. Key parameters value

look-up tables, which are validated based on the experiments. The high-voltage battery is based on a dual polarization circuit, whose parameters are tuned to be as close as possible to the real powertrain. The traction motor is loaded with the efficiencies and possible losses to mimic a realistic electric drive and the CVT module. Each of these components have their own low-level controllers, which will not be altered in this study. The supervisory controller determines the power split between the engine and the traction motor for propelling the vehicle, as well as CVT ratio.

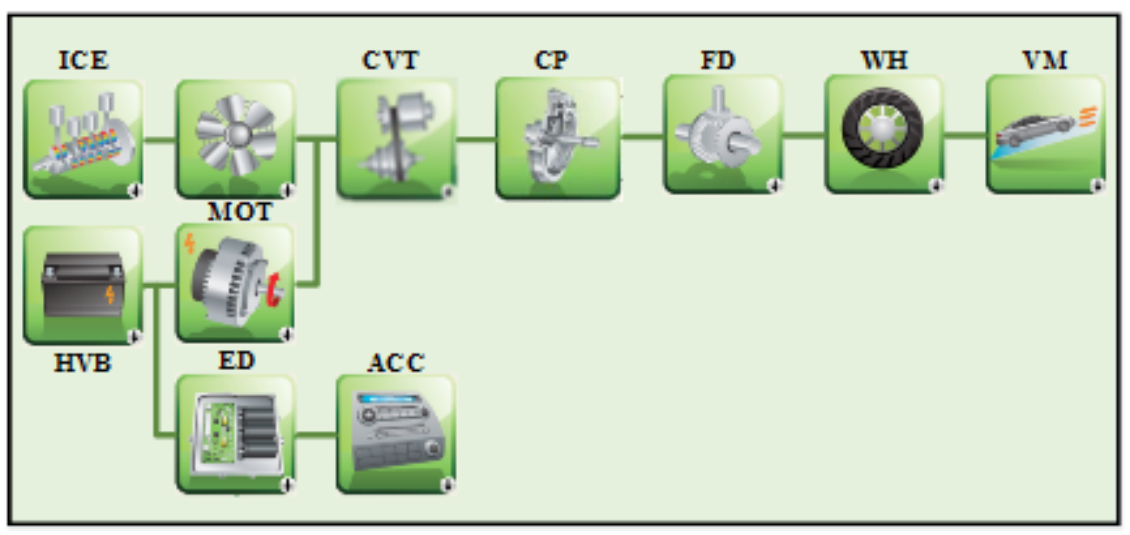

Fig. 1. Schematic of Autonomie high-fidelity model of a parallel HEV with CVT 
We will bypass the baseline high-level controller and connect the wires to our proposed controller.

\section{ENERGY MANAGEMENT SYSTEM}

Once the control-oriented model is obtained, the energy management system can be designed. The MPC approach has drawn a great deal of attention in among the relevant researchers and industries. MPC has an almost unique ability to handle hard constraints on control and states during its design procedure. It can predict the behaviour of the system at specified time intervals at the future instance via the controloriented model [17]. MPC merges the efficiency of an optimal control approach and the robustness of a feedback controller. However, the MPC approach has its own limits and challenges. The key drawback of this approach is its computational burden. However, the solver involved in solving the required optimization problem might be unreliable and bulky for naive control hardware in automotive applications. Those two are considered to be serious problems of MPC in real-time systems. Fortunately, there are solutions to those shortcomings. The computational burden can be alleviated by multi-parametric optimization approaches, which solve the problem offline and results in some affine functions of the parameters. Those functions' parameters can be stored on the hardware along with some look-up tables to represent the MPC solution to the problem. Although the nonlinear problems can be burdensome in this case, researchers can find an approximate solution instead. Another way to tackle the real-time related problems of MPC is to develop specific and simplified mathematical solvers to enjoy the inherent robustness of the online implementation. However, to maintain a small size of the controller and computational time, the former approach is selected.

In this research, the multi-parametric class of MPC is utilized and, to pursue the nonlinearity of the aforementioned control-oriented model, it is linearized for multiple operating point; in the end, a single controller is proposed. Consider the discrete and linear state-space model based on Eq. (2). For handling the tracking feature of the controller, we take the control-oriented model into the error space where the new state variables are the difference between $S O C$ and $V$, and their reference values as:

$$
\begin{aligned}
x(k+1) & =A x(k)+B u(k)+F, \\
y(k) & =C x(k),
\end{aligned}
$$

are the manipulated inputs, $y(k) \in R^{2}$ are the outputs. Let $x_{1}=e_{S O C}=S O C-S O C_{r e f}$ and $x_{2}=e_{V}=V-V_{\text {ref }}$. By considering prediction and control horizon, the prediction equation is obtained. In this equation, the inputs and outputs are:

$$
U=\left[\begin{array}{c}
u(k \mid k) \\
u(k+1 \mid k) \\
\vdots \\
u\left(k+N_{c}-1 \mid k\right)
\end{array}\right], \quad Y=\left[\begin{array}{c}
y(k+1 \mid k) \\
y(k+2 \mid k) \\
\vdots \\
y\left(k+N_{p} \mid k\right)
\end{array}\right]
$$

where $u\left(k_{i}+1 \mid k_{i}\right)$ means the predicted output at $k_{i}+1$ step based on the measurement at $k_{i}$ step, $N_{c}$ and $N_{p}$ are the control and prediction horizon length. By applying the input vectors to the system model, the predicted outputs are calculated and stored. In MPC control, optimal system inputs are calculated by minimizing a cost function. According to Eq. (5), the standard form of the cost function is:

$$
\begin{array}{ll}
\min & \frac{1}{2} U^{T} H U+q^{T} U+r \\
Z & \text { s.t } \quad G U \leq W
\end{array}
$$

$Z \in R^{s}, H=H^{T}>0 \in R^{s \times s}, W \in R^{m}, G \in R^{m \times s}, q \in R^{s}$.

If the objective function is convex, the local optimum point is the global optimum point. The optimal point can be found by solving this optimization problem. Finally, the cost function is formulated according to the expected control objectives. As mentioned before, the advantages of the MPC is the systematic handling of the system constraints. There are three major types of constraints frequently encountered in applications. The first two types deal with constraints imposed on the control variables $u(k)$, and the third type of constraint deals with output $y(k)$ or state variable $x(k)$ constraints [18]. The cost function for minimizing fuel consumption and improve drivability while meeting the hard constraints can be written as:

$$
J=\sum_{i=1}^{N_{p}} x_{i}^{T} Q_{x} x_{i}+\Delta x_{i}^{T} Q_{\Delta x} \Delta x_{i}+u_{i}^{T} R_{u} u_{i}+\Delta u_{i}^{T} R_{\Delta u} \Delta u_{i}, \text { (6) }
$$

where the regulation objective, minimum variation for the states., especially in terms of velocity, minimum effort and its variation are considered via the tuning matrices $Q_{x}, Q_{\Delta x}, R_{u}$, and $R_{\Delta u}$, respectively. This optimization problem is subjected to the following constraints, where the parameter values are shown in Table 1.

where $x(k) \in R^{2}$ are the state variables, $u(k) \in R^{4}$ 


$$
\begin{array}{rlrl}
T_{m, \text { min }} & <T_{m}<T_{m, \text { max }} & & \\
T_{\mathrm{e}, \text { min }} & <T_{e}<T_{\mathrm{e}, \max } & 0.2<S O C<1 \\
i_{c v t, \text { min }} & <i_{c v t}<i_{c v t, \max } & & 0<V . \\
T_{\mathrm{b}, \text { min }} & <T_{b}<T_{\mathrm{b}, \text { max }} & &
\end{array}
$$

The computational burden of the aforementioned problem might be an issue for real-time applications, because a constrained optimization problem should be solved at each time step. This requires capable and expensive hardware along with a reliable solver. To avoid those potential problems, we solve the MPC explicitly. In this way, we can find some regions throughout the state space. It can be shown that for a quadratic optimization problem with affine constraints, each region contains an affine function of the related parameters as the solution. In other words, the manipulated inputs are piecewise affine functions of state variables:

$$
u=\beta_{j} x+\gamma_{j},
$$

where $\beta_{j}$ and $\gamma_{j}$ are the constants for $j^{\text {th }}$ region.

Those constants are different for the regions and can be obtained by solving the MPC problem explicitly. The explicit MPC (eMPC) replaces the need for solving the optimization problem online with storing tables of the affine functions' coefficients and a point location procedure. This approach improves the real-time implementation capability of the controller. At the implementation stage, we need to have the initial state variable (parameter of eMPC) and then find the region that contains it (point location procedure). Once the region is found, the related coefficients $(\beta$ and $\gamma$ ) are available. To find the manipulated input, the initial state variable is substituted into Eq. (8) [19]. By scaling the state variables and manipulated inputs and solving the problem parametrically, the following regions are derived based on the multi-parametric toolbox (MPT) [20], as shown in Fig. 2. Inside each of 55 regions for prediction horizon with the length of 3 steps, there is an affine function of the parameters, namely, the difference between state variables and reference values. By increasing the prediction horizon length, we obtain more regions for better controller performance. However, this action increases the size of the controller, which might be problematic for the control hardware. In fact, there is a trade-off between simplicity and performance, and the design procedure should be directed in such a way that it addresses those two extremes efficiently.

Those affine functions are depicted in Fig. 3 for four manipulated inputs that are scaled based on their maximum values $\left(\bar{\alpha}=\alpha /\left|\alpha_{\max }\right|\right)$.

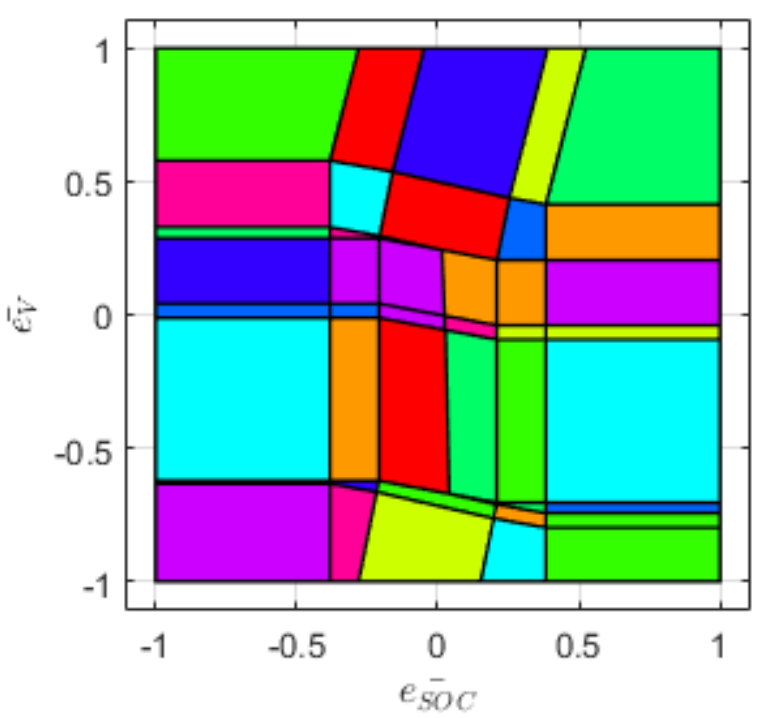

Fig. 2. Proposed EMS polytopes
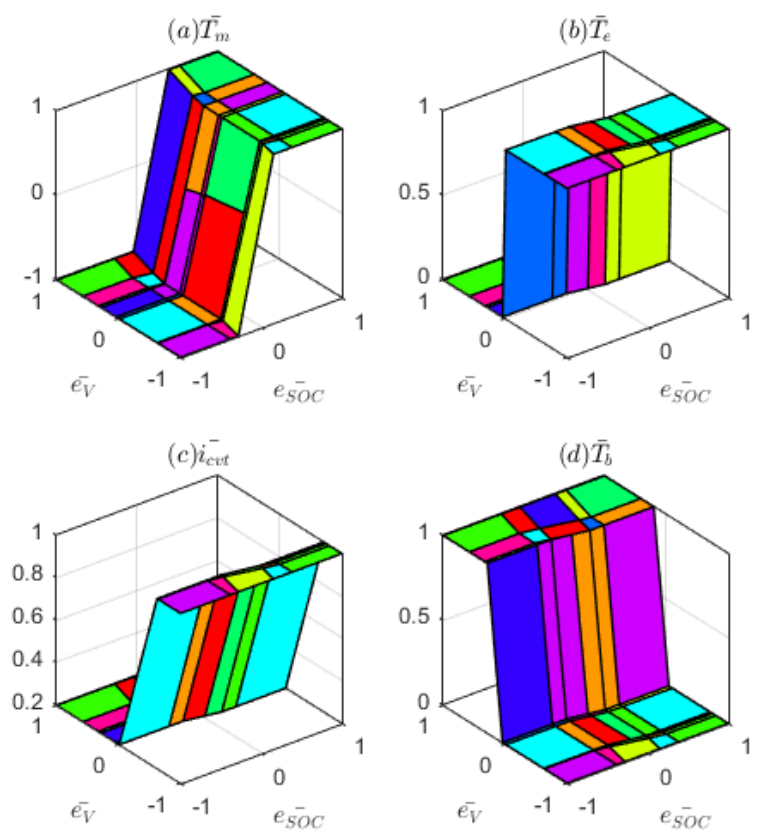

Fig. 3. Proposed EMS manipulated inputs vs parameters: scaled a) motor torque b) engine torque, c) CVT ratio, and

\section{d) braking torque}

As seen, we can divide the parameter regions into four sections.

1. $\bar{e}_{S O C}<0$ and $\bar{e}_{V}<0$ : In this region (which represents the toughest scenario for the EMS) the vehicle velocity is demanded to be increased and battery SOC is less than the predefined level. Therefore, the energy management system is not able to count on the electric drive to propel the vehicle. As a result, the braking torque should be 
zero, and the electric machine should be set into the generator mode (negative torque) to charge the battery. However, the ICE should be pushed to its limits so that it can propel the vehicle and charge the battery at the same time. Nevertheless, based on the difference between the desired and current velocity, the CVT ratio may vary between its limits. According to Fig. 3c, as the absolute value of $\bar{e}_{V}$ gets larger, a higher CVT ratio is required to increase the driving torque on the wheels so that the vehicle would reach the required speed more quickly.

2. $\bar{e}_{S O C}>0$ and $\bar{e}_{V}<0$ : Although this region resembles the above one, EMS faces a more gentle scenario since the battery state of charge is higher than its reference. Thus, the electric drive can assist the engine in propelling the vehicle. Braking torque is zero again, the engine torque and CVT ratio alter with the magnitude of $\bar{e}_{V}$, and the motor torque is positive and tops out it maximum value for more severe acceleration.

3. $\bar{e}_{S O C}<0$ and $\bar{e}_{V}>0$ : Here, EMS should slow down the vehicle since the current velocity is higher than its reference. In contrast, the SOC level should be increased. Therefore, there is a good chance for regenerative braking and, as shown in Fig. 3a, the electric drive switches to the generator mode. Moreover, the mechanical braking torque would no longer be zero.

4. $\bar{e}_{S O C}>0$ and $\bar{e}_{V}>0$ : In this region, the vehicle velocity should be decreased, and there is no need to charge the battery. As such, the engine is off, and the braking torque is high.

By reviewing the aforementioned regions, it turns out that the manipulated inputs trend matches the expectation from EMS. However, the shape of those surfaces determines the optimality and the performance of the controller. To investigate this, we need to implement those curves into the simulation high-fidelity model and check the overall performance over the standard driving schedules. Nonetheless, the convex cost function. as shown in Fig. 4, confirms the optimality of the produced controls scheme.

\section{CONTROL PROTOTYPING}

The HIL simulation is a more realistic platform to evaluate controller performance as opposed to the model-in-the-loop simulation. In a HIL platform, real signals from a controller are transferred to a test system that mimics reality. For this research, a dSPACE HIL platform has been used. In addition to the simulator, a

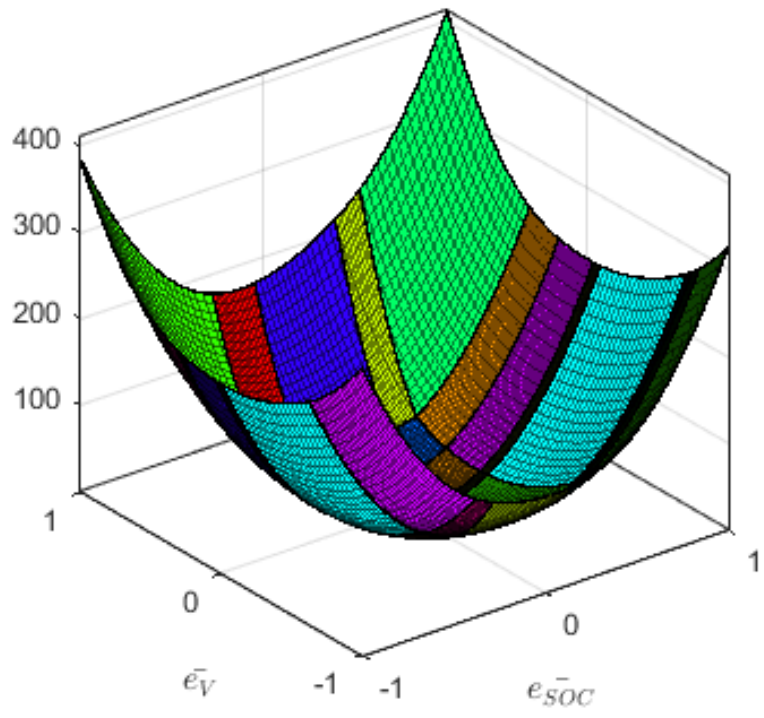

Fig. 4. Multi-parametric optimization optimal values

real-time model and prototype hardware are required to augment the controller capability. Fig. 5 depicts the schematic of the HIL test platform.

The virtual simulation model is accommodated to a real-time machine. The Autonomie model of the HEV is stored on the simulator (DS1006) and run in real time. In each time step, the simulator solves the high-fidelity model and sends back the required information to the control hardware via a controller area network (CAN). The most challenging part of an HIL test is to generate a C-code from the controller and to load it onto the control hardware. We considered this hindrance beforehand in the control design stage, at which point we solved the problem to obtain some polytopes along with some piecewise affine functions. Therefore, we face minimal effort to implement the controller onto the control hardware. A few specific libraries in dSPACE are allocated for generating a C-code for different devices, such as MicroAutoBoxII and DS1006 processor boards. As such, by using those functions, a C-code of the proposed control scheme is derived and loaded onto the control hardware (MicroAutoBoxII). For enabling communication with the simulator over the CAN bus, the MicroAutoBox has two bifurcated CAN controllers (four CAN channels) [21]. To facilitate this communication along with uploading the executable files, managing the test, and recording the simulation data, ControlDesk software is utilized.

The specifications of the input and output signals are determined in Table 2. The resulted polytopes of the controller are implemented into the 


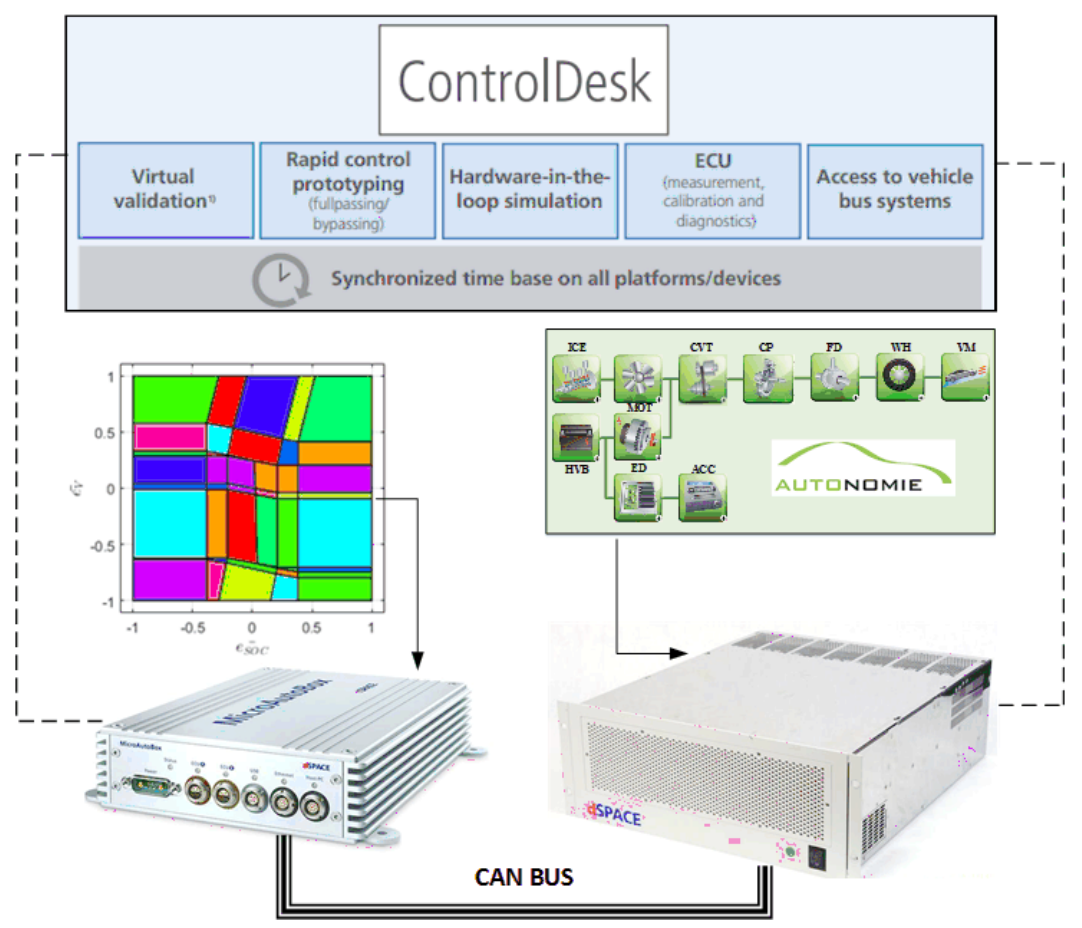

Fig. 5. dSPACE HIL testing platform schematic

MicroAutoBox. Basically, most of the ECU's physical memory is occupied by the CAN BUS communication section. Hence, there is a limited physical memory left to fit the controller logic. The total maximum occupied size of the proposed controller is barely around 100 $\mathrm{Kb}$, which is promising.

Table 2. HIL signals

\begin{tabular}{llcc}
\hline CAN Signal & Variable & CAN ID & Bit length \\
\hline \multirow{3}{*}{$\begin{array}{l}\text { MicroAutoBox } \\
\text { Input }\end{array}$} & SOC & 100 & 16 \\
\cline { 2 - 4 } & Reference SOC & 101 & 16 \\
\cline { 2 - 4 } & Vehicle velocity & 102 & 32 \\
\cline { 2 - 4 } & Desired vehicle velocity & 103 & 32 \\
\hline \multirow{3}{*}{$\begin{array}{l}\text { MicroAutoBox } \\
\text { Output }\end{array}$} & Motor torque & 120 & 32 \\
\cline { 2 - 4 } & Engine torque & 121 & 32 \\
\cline { 2 - 4 } & CVT ratio & 122 & 32 \\
\cline { 2 - 4 } & Braking torque & 123 & 32 \\
\hline
\end{tabular}

The proposed energy management system requires four readings from the real-time machine: the current battery state of charge, the reference SOC, vehicle velocity, and the desired velocity to find $e_{S O C}$ and $e_{V}$, respectively. The four measurements are done by the real-time machine using the Autonomie model introduced in Section 1.2. Now multi-parametric EMS looks for the appropriate motor torque, engine torque, CVT ratio and braking torque, which can be directly implemented to the Autonomie model. Moreover, the low-level controllers in the Autonomie model can make the engine and electric motor follow the transferred set points, which are dictated by MicroAutoBox.

Moreover, the controller is supposed to provide the appropriate control action within a period shorter than the model solver step size by considering CAN bus communication delays. Thus, the controller should be faster than the plant, which can be investigated through the HIL procedure. By performing the HIL test for different scenarios, it is observed that the turnaround time is less than 30 microseconds, which proves that the proposed EMS is much faster than real time. In the next section, we review the HIL simulation results.

\section{RESULTS AND DISCUSSIONS}

To validate the performance of the controller, we have considered three different driving schedules: the highway fuel economy test (HWFET), NEDC and the worldwide harmonized light vehicles test cycle (WLTC). To demonstrate the performance of the proposed approach, a comparison is made with the baseline Autonomie controller. Fig. 6 shows the simulation results for the HWFET cycle. As shown 
in Fig. 6a, the drivability performance remains satisfactory, since the controller tracks the velocity set point carefully. Although the final value of SOC for both approaches are the same, there is a difference between the battery SOC trajectory for the proposed controller and the baseline throughout the driving schedule. The proposed EMS has used the battery more frequently than the baseline controller in order to optimize the ultimate fuel economy of the vehicle. A part of this difference is due to the predictive nature of the proposed controller, as it has more knowledge about the future. Since there are some braking occasions ahead, the EMS allows the electric drive to deplete the battery more frequently as compared to the baseline strategy. Fig. $6 \mathrm{~b}$ depicts the CVT ratio, which results from the proposed EMS, in which the CVT ratio is lower than the baseline controller in total. Because of the highway and the high-speed schedule, the CVT is expected to be tuned into its lower ratios, which is confirmed in the plots. Fig. $6 \mathrm{c}$ and d show the engine power and the torque. The engine power for the proposed EMS is slightly lower than the baseline strategy because of the more frequent assistance of the electric drive, which contributes to better fuel
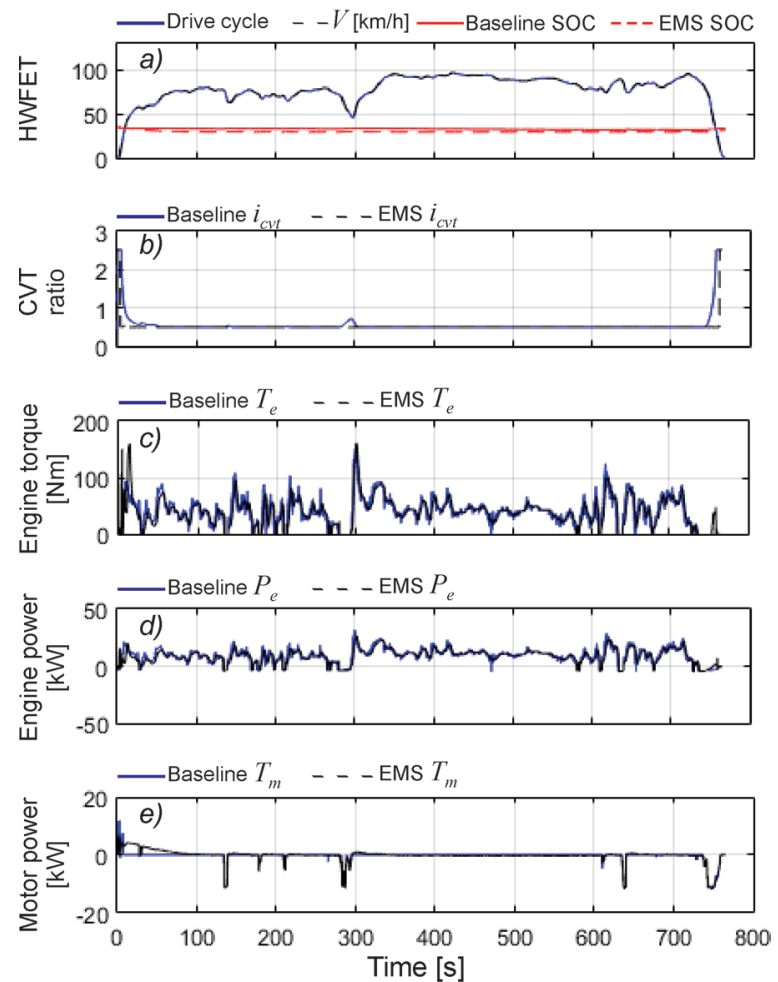

Fig. 6. HWFET cycle simulation; a) vehicle velocity $[\mathrm{km} / \mathrm{h}]$ and battery SOC, b) CVT ratio, c) engine torque, d) engine power, e) electric motor power economy. This is more visible at the beginning of the drive cycle, for which higher acceleration is demanded. Fig. 6e shows the electric drive power, which is negative for the braking parts of the drive cycle to recuperate a part of mechanical braking energy dissipation.

According to Fig. 7a, the drivability performance of the proposed controller for NEDC schedule is acceptable, but because of the pattern of the driving schedule, the resulted battery SOC for two controllers are close to each other. The major difference in the two controllers is shown in Fig. 7b, where the proposed EMS tends to keep the CVT ratio lower for better engine fuel economy. Consequently, lower engine torque is observed from implementing the baseline strategy in Fig. 7c. However, the total power of the engine throughout the drive cycle is lower for the proposed control scheme as in Fig. 7d. Fig. 7e shows the electric motor assistance especially for the citydriving microcycles.

Similarly, the drivability index is confirmed for the WLTC cycle according to Fig. 8a by implementing the proposed EMS. Once again, the proposed EMS results in a lower CVT ratio more frequently than
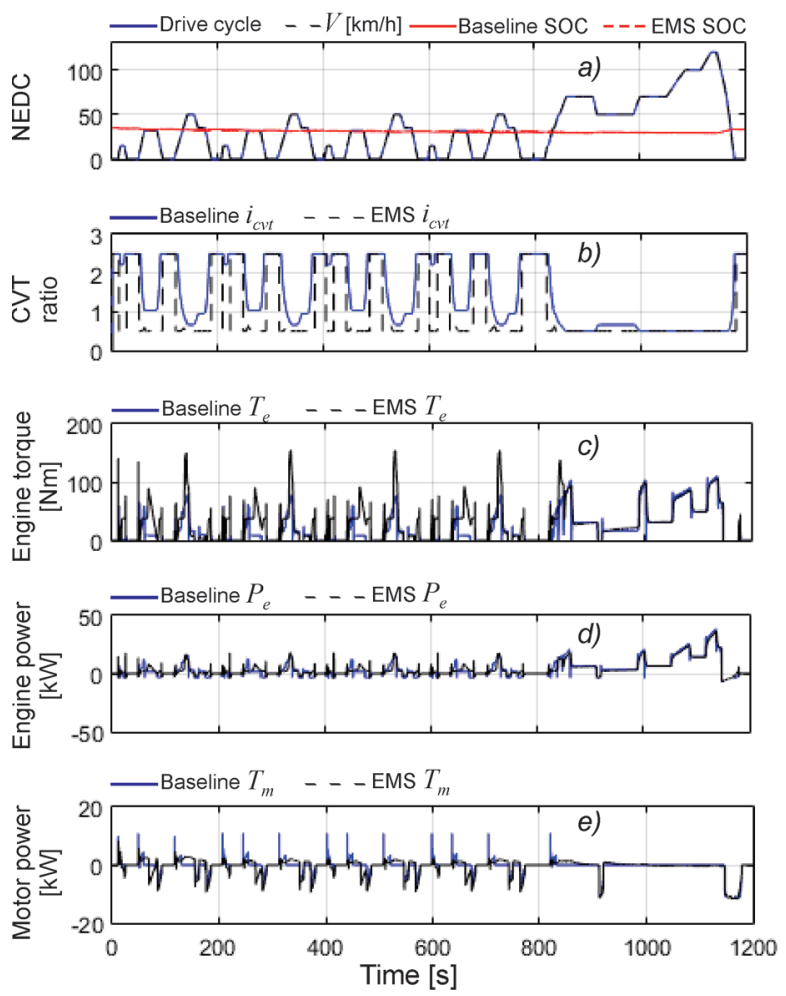

Fig. 7. NEDC cycle simulation; a) vehicle velocity $[\mathrm{km} / \mathrm{h}]$ and battery SOC, b) CVT ratio, c) engine torque, d) engine power, e) electric motor power 
the baseline controller in Fig. 8b. As such, the engine has better fuel economy despite operating in regions with higher torques (Fig. 8c). Slightly lower engine power and higher overall power of the electric drive in comparison to the baseline strategy proves the superior fuel economy of the proposed EMS, as in Figs. 8d and e.
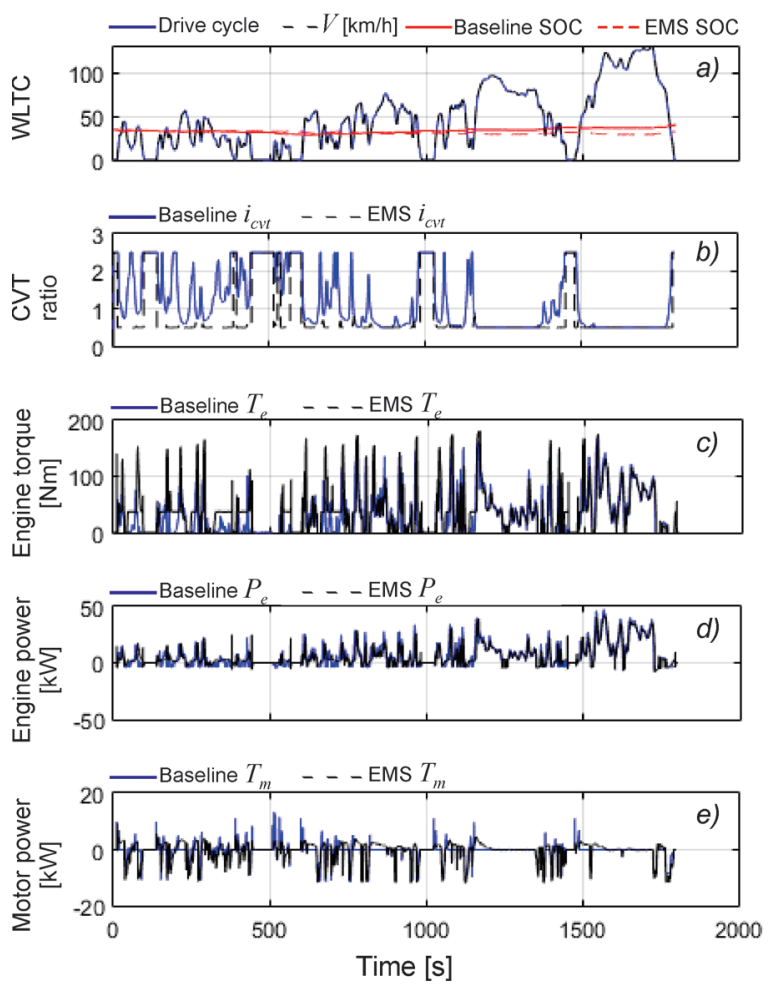

Fig. 8. WLTC-class 3 simulation; a) vehicle velocity $[\mathrm{km} / \mathrm{h}]$ and battery SOC, b) CVT ratio, c) engine torque, d) engine power, e) electric motor power

Based on the travelling distance associated with each of the drive cycles, the fuel economy for the baseline Autonomie controller and the proposed EMS is calculated in Table 3.

Table 3. Fuel economy values

\begin{tabular}{lcccc}
\hline Drive & $\begin{array}{c}\text { Traveling } \\
\text { cycle }\end{array}$ & \multicolumn{2}{c}{ Fuel economy [l/100 km] } & Improvement \\
\cline { 3 - 4 } & distance $[\mathrm{km}]$ & Baseline & Proposed EMS & {$[\%]$} \\
\hline HWFET & 16.450 & 4.988 & 4.796 & 4 \\
\hline NEDC & 11.000 & 5.788 & 5.116 & 11.61 \\
\hline WLTC & 23.194 & 6.083 & 5.442 & 10.54 \\
\hline
\end{tabular}

The more realistic Autonomie high-fidelity model ensures the presentation of real-world results for implementing the proposed EMS. As expected for HWFET, the fuel economy improvement for the higher vehicle velocity is limited as the engine is more efficient and experienced less transience, so that little assistance is required from the electric drive. For the mixed city and highway driving of NEDC and WLTC cycles, the proposed EMS results in about 11 per cent lower fuel consumption in comparison to the baseline strategy. To draw a more comprehensive comparison, similar studies are considered as well. Tang et al. [13] have obtained a similar fuel economy for NEDC cycle using a Gauss pseudo-spectral MPC to reduce the computational time for the controller on the same architecture of hybrid vehicle with close parameter values to our study. However, the proposed EMS in this study is completely real-time implementable and downsized for a commercial control hardware. Moreover, its performance and capability are validated with HIL testing via the Autonomie simulation model.

\section{CONCLUSIONS}

In this research, a real-time implementable multiparametric predictive energy management system is proposed for parallel hybrid electric vehicles with CVT driveline. To exploit the accuracy of the powertrain model and maintain its simplicity as appropriate for a model-based control approach, a control-oriented model is considered based on the vehicle velocity and onboard high-voltage battery. To investigate the performance of the controller, a hardware-in-the-loop simulation via Autonomie experimentally-validated model of the powertrain is performed. The results of applying the sub-optimal predictive EMS to the mentioned simulation model shows more than $8 \%$ improvement in fuel economy for three standard driving schedules. The proposed EMS can be fully-implemented in control hardware for real-time applications.

\section{ACKNOWLEDGMENT}

The authors acknowledge the Smart Hybrid and Electric Vehicles Systems (SHEV) lab at the University of Waterloo for providing them with the required platform for performing the simulations.

\section{REFERENCES}

[1] Pfiffner, R., Guzzella, L. (2001). Optimal operation of CVTbased powertrains. International Journal of Robust Nonlinear Control, vol. 11, no. 11, p. 1003-1021, Dol:10.1002/rnc.642.

[2] Hatanaka, T., Yamada, T., Fujita, M., Morimoto, S., Okamoto, M. (2009). Explicit receding horizon control of automobiles with continuously variable transmissions. Nonlinear Model 
Predictive Control, vol. 384, p. 561-569, D0I:10.1007/978-3642-01094-1_46.

[3] Kazemi, R., Raf'at, M., Noruzi, A.R. (2014). Nonlinear optimal control of continuously variable transmission powertrain. International Scolary Research Notices, vol. 2014, art. ID 479590, Dol:10.1155/2014/479590.

[4] Yildiz, A., Kopmaz, O. (2017). Control-oriented modelling with experimental verification and design of the appropriate gains of a PI speed ratio controller of chain CVTs. Strojniški vestnikJournal of Mechanical Engineering, vol. 63, no .6, p. 374-382, DOl:10.5545/sv-jme.2016.4184.

[5] Bemporad, A., Bernardini, D., Livshiz, M., Pattipati, B. (2018). Supervisory model predictive control of a powertrain with a continuously variable transmission. SAE Technical Paper, DOI:10.4271/2018-01-0860.

[6] Tao, D., Zhifei, L., Yanli, Y. (2019). Research on the novel discretized ratio control strategies and hardware-in-the-loop test for continuous variable transmission system. International Journal of Modeling, Simulation, and Scientific Computing, vol. 10, no. 3, D0l:10.1142/S1793962319500120.

[7] Mamala, J., Jantos, J. (2009). Shift speed control in CVT powertrain. International Journal of Vehicle Design, vol. 54, no. 1, p. 26-34, D0I:10.1504/IJVD.2010.034868.

[8] Takiyama, T., Morita, S. (1999). Engine-CVT consolidated control using LQI control theory. JSAE Review, vol. 20, no. 2, p. 251-258, DOI:10.1016/S0389-4304(98)00062-9.

[9] Kim, T., Kim, H., Yi, J., Cho, H. (2000). Ratio control of metal belt CVT. SAE Technical Paper, D0l:10.4271/2000-01-0842.

[10] Hu, J., Mei, B., Peng, H., Guo, Z. (2019). Discretely variable speed ratio control strategy for continuously variable transmission system considering hydraulic energy loss. Energy, vol. 180, p. 714-727, D0I:10.1016/j.energy.2019.05.086.

[11] Hofman, T., Steinbuch, M., van Druten, R., Serrarens, A.F.A. (2009). Design of CVT-based hybrid passenger cars. IEEE Transactions on Vehicular Technology, vol. 58, no. 2, p. 572587, D0I:10.1109/TVT.2008.926217.

[12] Ji, J., Park, J., Kwon, O., Kim, H. (2012). An energy management strategy for a CVT based parallel hybrid electric vehicle. IEEE Vehicle Power and Propulsion Conference, $\mathrm{p}$. 380-382, D0I:10.1109/VPPC.2012.6422668.

[13] Tang, J., Guo, L., Gao, B., Liu, Q., Yu, S., Chen, H. (2016). Energy management of a parallel hybrid electric vehicle with CVT using model predictive control. 35th Chinese Control Conference (CCC), p. 4396-4401, D0l:10.1109/ Chicc.2016.7554036.

[14] Li, H., Hu, X., Fu, B., Wang, J., Zhang, F., Zhou, Y. (2019). Effective optimal control strategy for hybrid electric vehicle with continuously variable transmission. Advances in Mechanical Engineering, vol. 11, no. 3, D0l:10.1177/1687814018824811.

[15] Li, H., Zhou, Y., Xiong, H., Fu, B., Huang, Z. (2014). Realtime control strategy for cvt-based hybrid electric vehicles considering drivability constraints. Applied Sciences, vol. 9, no. 10, art. ID 2074, D0I:10.3390/app9102074.

[16] Wang, Q., Frank, A.A., (2014). Plug-in HEV with CVT: configuration, control, and its concurrent multi-objective optimization by evolutionary algorithm. International Journal of Automotive Technology, vol. 15, p. 103-115, D0l:10.1007/ s12239-014-0012-z.

[17] Del Re, L., Allgöwer, F., Glielmo, L., Guardiola, C., Kolmanovsky, I. (eds.) (2010). Automotive Model Predictive Control: Models, Methods and Applications, Springer, London, DOl:10.1007/978-1-84996-071-7.

[18] Wang, L. (2009). Model Predictive Control System Design and Implementation Using MATLAB®. Springer, London, DOl:10.1007/978-1-84882-331-0.

[19] Taghavipour, A., Azad, N.L., McPhee, J. (2015). Real-time predictive control strategy for a plug-in hybrid electric powertrain. Mechatronics, vol. 29, p. 13-27, D0l:10.1016/j. mechatronics.2015.04.020.

[20] Herceg, M., Kvasnica, M., Jones, C.N., Morari, M. (2013). Multiparametric toolbox 3.0. Proceedings of the European Control Conference, p. 502-510, D0I:10.23919/ECC.2013.6669862.

[21] Taghavipour, A., Vajedi, M., Azad, N.L. (2019). Intelligent Control of Connected Plug-In Hybrid Electric Vehicles. Springer, Cham, Dol:10.1007/978-3-030-00314-2. 